\title{
HYPERSET/WEB-LIKE DATABASES AND THE EXPERIMENTAL IMPLEMENTATION OF THE QUERY LANGUAGE DELTA Current State of Affairs
}

\author{
Richard Molyneux, Vladimir Sazonov \\ Department of Computer Science, University of Liverpool, Ashton Street, Liverpool, United Kingdom \\ molyneux@csc.liv.ac.uk,sazonov@csc.liv.ac.uk
}

Keywords: Web-like, semistructured, distributed databases, hypersets, bisimulation, query language Delta

Abstract: $\quad$ The hyperset approach to WEB-like or semistructured databases is outlined. WDB is presented either (i) as a finite edge-labelled graph or, equivalently, (ii) as system of (hyper)set equations or (iii) in a special XML-WDB format convenient both for distributed WDB and for including arbitrary XML elements in this framework. The current state of affairs on experimental implementation of a query language $\Delta$ (Delta) to such databases-the main result of this paper-is described, with consideration of further implementation work to be done.

\section{INTRODUCTION}

It appears that the great success of Codd's relational approach to databases (Codd, 1983) was based on taking the most fundamental concepts of logic and set theory as its foundation. Thus, any relation is a set of tuples, each tuple being also represented by a set of a special kind. From the second half of the 1990s a new idea of semistructured databases (SSDB) emerged; see (Abiteboul et al., 2000) as a general reference. In the age of the Internet and the WorldWide Web, allowing accessibility of remote and heterogeneous databases, the relational paradigm has become too narrow and restrictive. The structure of the data over the Internet is typically non-fixed or nonuniform. The idea of graph representation of data was introduced with interpretation of graph (directed) edges like links to browse the Web. Furthermore, because of such "browsing", considering the graph as a binary (or ternary, if taking labels on edges into account) relation is not fully adequate in this context. That is, intuitively this is something more than just a relation. Indeed, interpreting end nodes of outgoing edges from any given node $n$ as "children" or even as "elements" of $n$ becomes more appropriate. In particular, the latter is the terminology of XMLthe widely adopted approach to semistructured data (however, tree-like rather than graph-like).

This also leads us again to a set theoretic idea of representation of data-now semistructured data-a far going generalization of the relational approach. It is also worth noting that in the foundations of mathematics, the previous century was marked by the triumph of the set theoretic approach for representating mathematical data (the concepts) as well as the style of mathematical language and reasoning. Mathematical logicians also developed generalized computability theory over abstract sets (of sets of sets, etc.) in the form of admissible set theory (Barwise, 1975). In computer science, a set theoretic programming language SETL was created (quite naturally, for the case of finite sets only). Also some theoretical considerations on computability and query languages over hereditarily finite sets were done in (Dahlhaus and Makowsky, 1986; Dahlhaus and Makowsky, 1992; Sazonov, 1987; Sazonov, 1993) with the perspective of a generalized set-theoretically presented databases-in fact semistructured oneseven before the term "semistructured databases" had arisen. Moreover, the set theoretic approach is closely related with a special version of the graph approach. Probably the first mathematical result relating both approaches was Mostowski's Collapsing Lemma allowing to interpret graph nodes as sets of sets corresponding to children of these nodes. This, however, worked properly only for well-founded graphs and sets (which in the finite case, especially interesting for database applications, means the absence of cy- 
cles). But arbitrary graphs, even with cycles can also be "collapsed" into sets (interrelated by the membership relation) in the more general non-well-founded set theory called also hyperset theory (Aczel, 1988; Barwise and Moss, 1996). Here, for example the set $\Omega=\{\Omega\}$ consisting of itself and corresponding to the loop graph $\circlearrowleft$ is quite a legal and meaningful thing.

These two trends (from set theory to semistructured databases as graphs and vice versa) were called in (Sazonov, 2006) top-down and bottom-up approaches to semistructured databases. They meet most closely in the work (Buneman et al., 2000) which is devoted to a specific graph approach to semistructured data considered up to bisimulation. The latter concept is the key one in (Lisitsa and Sazonov, 1997; Lisitsa and Sazonov, 1999) for interpreting graph nodes as a system of hypersets belonging one to another according to the graph edges. Nevertheless, (Buneman et al., 2000) is still rather a graph approach than hyperset one according to the special, however related to, but not a genuine set theoretical way as graphs are treated there; cf. (Sazonov, 2006).

Note that the alternative and popular XML representation of semistructured databases besides being based mainly on the idea of a finite tree (not arbitrary graph), assumes a fixed order on the children of any node, and therefore can not be considered as a (hyper)set approach. Also, any graph representation like in (Abiteboul et al., 1997; McHugh et al., 1997) which does not assume considering graphs up to bisimulation (to thereby make the nodes into abstract hypersets) is outside of the hyperset view.

The goal of this paper is to demonstrate how theoretical ideas of hyperset approach to semistructured (which can be also naturally called Web-like) databases (WDB) could be implemented and potentially applied in the form of a practically working query language Delta to such data. More precisely, we want to demonstrate that this is working in principle. The real practical work on efficiency as well as many other questions which should be resolved for any realistic database management system should be inevitably postponed here because this is rather work for a team of developers. However, we will present here some related considerations.

Organizing the paper. Section 2 is devoted to the background of the hyperset view to SSD/WDB with the details of how it is represented in terms of systems of set equations. Also an appropriately restricted XML (XML-WDB) format is introduced to represent set equations and to make this approach easily adapted to already widely accepted XML. It is assumed, in fact, a distributed version of a hyperset based WDB somewhat similar to, but not necessarily so huge as the WWW. Section 3 introduces the theoretical version of the language Delta and gives quick outline of the implemented version of Delta by examples of queries. Section 4 describes how such queries can be executed, again by examples. Section 5 outlines what should be done to make this language more practically applicable and efficient. We conclude in Section 6 by making some comparisons.

\section{HYPERSET APPROACH TO WDB}

The set theoretic and, more generally, hyperset approach (Sazonov, 1993; Lisitsa and Sazonov, 1997; Leontjev and Sazonov, 2001; Sazonov, 2001) to WDB is based on the concept of hereditary finite sets or more generally on hyperset theory (Aczel, 1988; Barwise and Moss, 1996). Such semi-structured data is represented as abstract sets of sets of sets, etc., even with the possibility of cycles. For visualisation purposes, hyperset databases are represented by graphs (as depicted in Figure 1) with directed edges representing the membership relations.

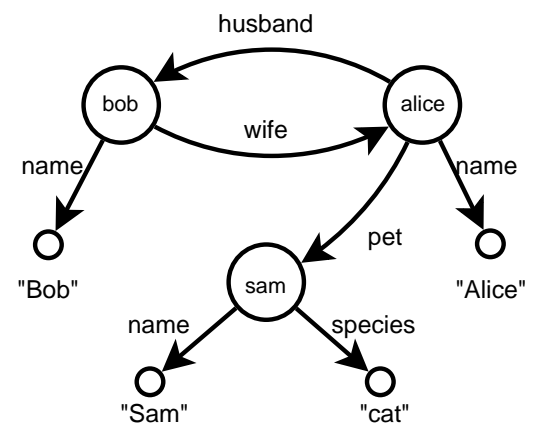

Figure 1: Example WDB representing a fictitious family

However, when considering implementation (and also intuitively) it is far more appropriate to represent WDB by corresponding system of set equations. Each set equation consists of a set name equated to the corresponding bracket expression, were labelled elements (of the set) may be either atomic values or nested bracket expressions or set names described in other equations. For example, the system of "unnested" or "flat" set equations generated by the graph in Figure 1 (and vice versa) looks as follows:

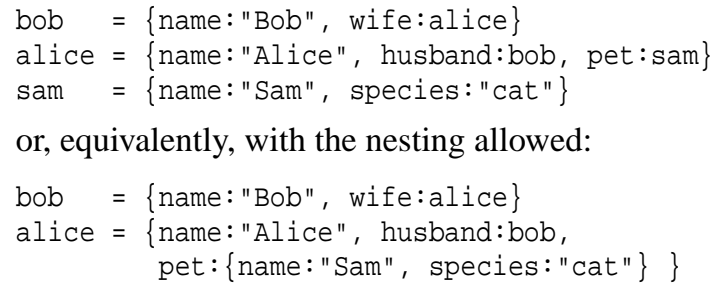


In particular, this demonstrates that the specific form of set names bob, alice, sam however helpful intuitively is formally not important. They can always be renamed (say, by numbered "object identities" like $\& 23$, etc.) or substituted as above. The proper information on the WDB is carried by (i) labels on WDBgraph edges (name, wife, husband, etc.), (ii) atomic data on leaves ("Bob", "Alice"), and (iii) the graph structure or, respectively set-element nesting.

Note 1 Atomic data is in fact treated as labels on additional leaf edges or, equivalently, as singleton sets consisting of a (labelled) empty set $($ "Bob" = $\{$ Bob: \{\}$\})$.

\subsection{Bisimulation}

In contrast to XML, the order and repetition of the elements in set equations play no role. This leads to the well-known (see e.g. (Aczel, 1988)) concept of bisimulation relation between graph nodes or set names $\left(n_{1} \approx n_{2}\right)$. This relation (and corresponding recursive algorithm) is based on the idea that any two sets are equal (or two set names and corresponding graph nodes are bisimilar) if for each (labelled) element of the first set there exists an equal (bisimilar) element in the second set, and vice-versa. Bisimilar set names are said to denote the same abstract (hyper)set. Thus, we are considering WDB-graph (or system of set equations) up to bisimulation which makes this approach more than just a pure graph theoretic one. WDB is called strongly extensional (Aczel, 1988) or non-redundant if different nodes (set names) are nonbisimilar i.e. denote different hypersets. This also means that $\approx$ coincides with $=$.

\subsection{WDB and XML}

Although set equations represent WDB in the most natural and intuitive way directly suggesting that our data are hypersets, it makes sense to relate this approach to the popular XML representation of semistructured data and probably to use appropriate existing techniques. Also numerous existing XML data can be treated by our approach what can make its application range considerably wider.

The main idea of the XML representation of any system of set equations consists in (recursive) replacing any labelled bracket expression label : $\{\ldots\}$ by XML element $<$ label $>\ldots</$ label $>$. More precisely, the nested version of the system of set equations above (for Figure 1) is represented as an XML document in the following special $X M L-W D B$ format:

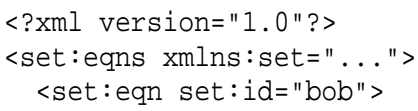

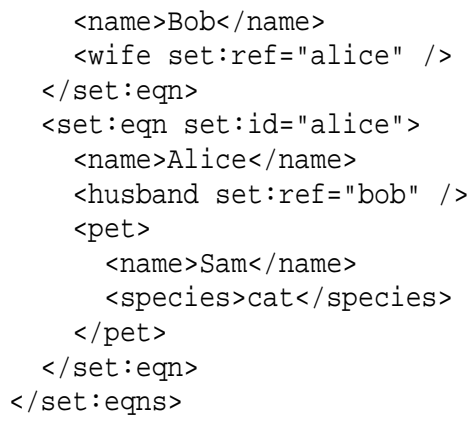

Here set:id is the required attribute of set:eqn element and should have a unique (across the whole document) value called also set name. Any other attributes, except set:ref, are also allowed in the element set:eqn. The attribute set:ref must refer to some existing set:id (possibly multiple-having the attribute type IDREFS). The elements set:eqn are allowed to contain arbitrary XML sub-elements with arbitrary attributes (except set : id) and text data.

Such a XML-WDB document can be treated as a system of set equations by using the following simple transformations which can be done in arbitrary order:

- <set:eqn set:id="setname">..</set: eqn> is replaced by equation setname $=\{\ldots\}$.

- any attribute (say, with two values) attr = "value1 value2", except the distinguished attributes set:id and set:ref, is removed and replaced by sub-elements

<attr>value $1</$ att $r><$ att $r>$ value $2</$ attr $>$. Thus, attributes are treated as tags.

- any text data transforms to the sequence of empty sub-elements $<$ any $/><$ text $/\rangle<$ data $/>$.

As we intend to ignore any order and repetitions (in contrast with the ordinary XML approach) this, in fact, means that a sentence (any text data) is considered rather as an unordered set of words.

- <tag $>\ldots</$ tag $>$ transforms to $\operatorname{tag}:\{\ldots\}$ and $<\operatorname{tag} />$ transforms to tag: \{\} .

- <tag set:ref="setname1 setname2 ..."/> transforms to the sequence tag: setname1, tag:setname2, ....

In the case if the element $<$ tag $>$ is non-empty with some content "...", as in the previous clause, we just add tag: $\{\ldots\}$ to the above sequence.

- Finally, omit < ?xml> and <syseqn> tags.

This way any XML-WDB document with arbitrary nested XML elements represents system of set equations with accordingly nested right-hand sides. In particular, arbitrary XML documents can be incorporated into our hyperset WDB framework (by nesting inside set: eqn elements). Both transformations from 
XML-WDB to system of set equations and vice versa have been implemented.

\subsection{Distributed WDB}

WDB system of set equations may be divided into several subsystems (XML-WDB files) with the possibility for the set names $s$ participating in one subsystem to be defined by set equations $s=\{\ldots\}$ in some other subsystems. To this end we introduce one more attribute set:href (in the same namespace) whose value should be full set name consisting of a URL of a possibly remote XML-WDB file concatenated with \#simple-set-name where simple-set-name is the set:id value from this file. So, strictly speaking, we should always assume full set names, even if using simple set names in set equations of distributed WDB. This also allows to avoid non-intended simple set name clashes in different XML-WDB files created possibly by various users in remote sites. It is required that each full set name should refer to an existing XML-WDB file and corresponding equation within that file for the simple set name part (after \# symbol). We also have an appropriate XML schema (Delta-WDB Site, 2007) for the XML-WDB format. Of course, the above transformation rules from XMLWDB to systems of set equations should be amended accordingly for set:href attribute. Anyway, this will lead to a (possibly huge) unified system of set equations-distributed WDB.

The analogy of WDB with the WWW does not imply it is so huge. It could be distributed between several sites and supported by specialized WDBservers of some branches (departments) of an organization. Another point is that WDB might be much more structured than the WWW, however the general approach imposes no restrictions. Nevertheless, the concept of WDB-schema (much more flexible than for the relational case and based on the concept of a bisimulation or "one-way" simulation) relativized to some typing relation on labels/atomic values can be considered for such databases ((Sazonov, 1993; Lisitsa and Sazonov, 1997) and e.g. (Abiteboul et al., 2000)).

As relational databases are (theoretically) a partial case of this hyperset approach, it should be straightforward to incorporate them into a WDB however heterogeneous they might be.

\section{QUERY LANGUAGE}

There has already been much theoretical considerations on (some versions of) the query language $\Delta$
(Delta) to hyperset/WDB databases. The two main syntactical categories of Delta are:

- $\Delta$-terms representing set valued operations over hypersets (set queries); and

- $\Delta$-formulas representing truth valued operations (boolean queries).

Inclusion of set theoretic operators of transitive closure (TC), recursion (Rec), and decoration (Dec) allows to define in $\Delta$ exactly all polynomial time computable operations over hypersets (represented as WDB), thus demonstrating and characterising theoretically its rich expressive power (assuming that a linear order on labels is given). The operators of $\Delta$ are defined as follows:

$$
\begin{aligned}
& \langle\Delta \text {-term }\rangle::=\langle\text { set variable or constant }\rangle \mid \emptyset \\
& \quad\left|\left\{l_{1}: a_{1}, \ldots, l_{n}, a_{n}\right\}\right| \bigcup a|\operatorname{TC}(a)| \operatorname{Dec}(a, b) \\
& \mid\{l: t(x, l) \mid l: x \in a \& \varphi(x, l)\} \\
& \quad \mid \operatorname{Rec} p .\{l: x \in a \mid \varphi(x, l, p)\} \\
& \langle\Delta \text {-formula }\rangle::=a=b\left|l_{1}=l_{2}\right| l_{1}<l_{2} \mid l_{1} R l_{2} \\
& \quad|l: a \in b| \varphi \& \psi|\varphi \vee \psi| \neg \varphi \\
& |\forall l: x \in a . \varphi(x, l)| \exists l: x \in a . \varphi(x, l)
\end{aligned}
$$

The intuitive set theoretic semantics of the majority of the above constructs should be well-understood by anybody with the minimal mathematical background in set theory and logic; see also (Sazonov, 2006). General note: $a, b, \ldots$ denote (set valued) terms, $x, y, z, \ldots$ are set variables and $l, l_{i}$ are label values (just strings of symbols) or variables, depending on the context, and $\varphi, \psi$ are (boolean valued) $\Delta$ formulas. Additionally, the binding label and set variables $l, x, p$ of quantifiers, collect, and recursion constructs above should not appear free in the bounding term $a$ (denoting a finite set) otherwise these operators become unbounded and thus, in general, noncomputable. Equality $=$ and the (alphabetic) ordering $<$ on labels is understood standardly. The relation $R$ on labels is any (easily) computable relation, in particular "to be a (prefix/infix/suffix) substring"-quite usable in queries. It could be also a relation : : of typing. For example we could have John: : name and June: :month. On the other hand, the equality between $\Delta$ terms/hypersets $a=b$ (or $a \approx b$ ) is understood as the equality of abstract hypersets denoted by these terms and, eventually, is computed by the bisimulation algorithm discussed above. Moreover, bisimulation is, in fact, implicitly involved in the (computational) meaning of the membership relation according to the equivalence

$$
l: a \in b \Longleftrightarrow \exists m: x \in b .(m=l \& x \approx a) .
$$

This means: find an outgoing $l$-labelled edge from $b$ which leads to some node $x$ bisimilar to $a$. But think- 
ing abstractly, $l: a \in b$ says just that $a$ is an $l$-labelled element of $b$. The recursion operator $\operatorname{Rec} p \cdot\{l: x \in$ $a \mid \varphi(x, l, p)\}$ defines a subset $\pi$ of the set denoted by $a$ obtained as the result of stabilizing (due to finiteness of $a$ ) the monotonic sequence of subsets of $a$ defined iteratively as $p_{0}=\emptyset$ and $p_{k+1}=p_{k} \cup\{l: x \in a \mid$ $\left.\varphi\left(x, l, p_{k}\right)\right\}$. The transitive closure $\mathrm{TC}(a)$ denotes the set of (labelled) elements of elements ... of elements of $a$. We refer to (Aczel, 1988) for the precise definition of the decoration operator Dec and only note here that it is the only operator in $\Delta$ which allows to construct real (cyclic) hypersets like $\Omega=\{\Omega\}$ or like in the Figure 1 from the ordinary "uncycled" sets (of sets of sets,...) of finite depth. This can be also reasonably called the plan performance operator (Sazonov, 2006) because its input(s) can be considered as a plan of construction of a hyperset, and the output is the resulting abstract hyperset. Imagine that we have a plan of a Web site (i.e. of a system of hyperlinked Web pages) and that Dec is a tool (a query) which automatically creates all the required Web pages.

Practically, constructs of the $\Delta$-query language are expressed as 'english-like' statements in block structured query language similar to SQL. Additional features have been (and even more are intended to be) added to $\Delta$ making the language more practically convenient, but not increasing its theoretical expressive power. Say, the powerset operation $\operatorname{Pow}(a)$ giving the set of all subsets of $a$ is evidently intractable (requiring exponential time and space) and is not worth to be added. But everything which is polynomial time computable is already definable in the original theoretical version of $\Delta$ presented above. Therefore, any additions we intend to make, however important practically, are just "syntactic sugaring" of the above theoretical version of $\Delta$. In particular, let us note the following extensions:

Library functions: Creation, deletion and modification of user defined/predefined queries and constants. These queries/constants can then be used in any query thus saving time and effort.

Queries with declarations: Similar to the library function but queries/constants are defined for (possibly multiple) use within a particular query.

For example, our BNF of $\Delta$ (Delta-WDB Site, 2007) contains the following production rules:

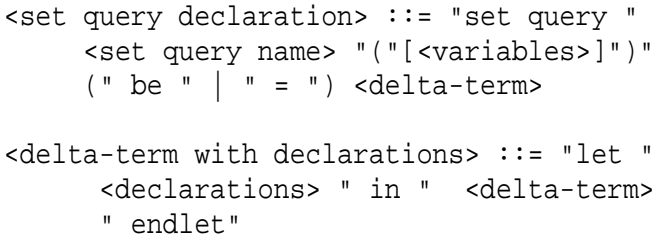

Analogously (and even simpler), set constant declarations are quite convenient. Recall that full set names are typically quite long, having the form url\#s imple-set-name; thus declaring a set constant, say $c$, for this set name will make queries much more readable and manageable.

\subsection{Syntactical Correctness and the Contextual Analysis}

Queries satisfying the BNF are called well-formed. But they should also be well-typed, with all identifiers properly declared or quantified The latter is achieved by tracking and suitably correcting the syntactical categories and types in the parse tree of a query. This guarantees that all identifiers are properly declared and typed (if this is possible at all). Occurrences of set or label variables or constants, the type of query declaration and corresponding query calls should mutually agree, etc.

\subsection{Example of non-Well-Typed Query}

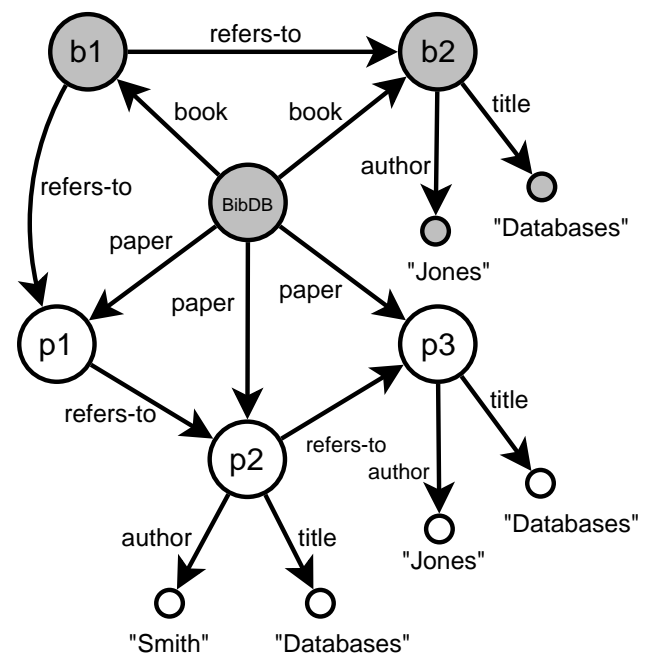

Figure 2: Example of a distributed-according to the colouring - bibliography WDB (similar to one from (Abiteboul et al., 2000)) represented as two XML-WDB files with URL1 and URL2; see (Delta-WDB Site, 2007).

The following is an attempt to query the bibliography WDB from Figure 2.

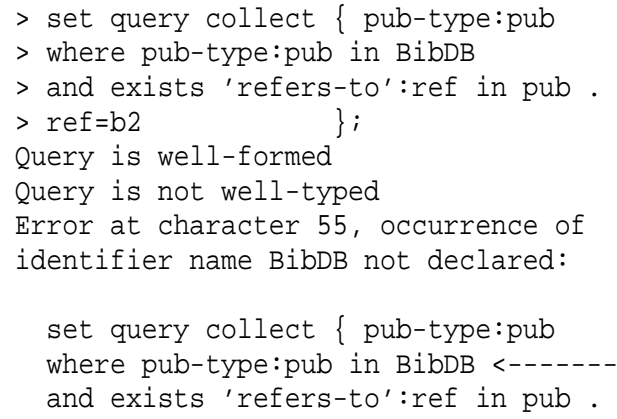


Error at character 104, occurrence of

identifier name b2 not declared:

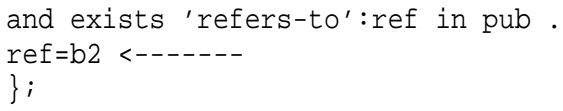

Note that $\mathrm{BibDB}$ and $\mathrm{b} 2$ are identifiers which are nowhere declared in this query. See the corrected version of this example in Section 4.1 where these identifiers are appropriately related to the WDB considered.

\section{QUERY EXECUTION}

To execute a (set or boolean) well-formed and welltyped query $q$ whose all participating set names (constants) are taken from the given WDB-a system of set equations-we should:

extend this system by new equation res $=q$ with res a fresh (i.e. unused in WDB) set or boolean, if such is $q$, name and

simplify the extended system

$$
\mathrm{WDB}^{\prime}=\mathrm{WDB}+(\text { res }=q)
$$

until it will contain only (possibly nested) bracket expressions as the right-hand sides of the equations or the truth values true or false (if the lefthand side is boolean name).

Thus, after simplification is complete, no complex set or boolean queries in set equations will appear, and the resulting version WDB-RES of WDB will consist, alongside with the old equations of the original WDB, of new set equations (new set names equated to possibly nested bracket expressions) and boolean equations (boolean names equated to boolean values true or false). This process is quite natural. For example, if the given query contains some complex subquery like $q=\bigcup q 1$ then the equation res $=q$ is split into two subqueries res $=\bigcup$ res 1 and $\operatorname{res} 1=q 1$ with res 1 a fresh set name. We omit further details referring the reader to (Sazonov, 2006). The point is that at the end we will have the equation res $=\{\ldots\}$ of the required form whose right-hand side may use some set names either from the original WDB or newly introduced during this process auxiliary set names (like res 1 above) with corresponding equations of the required form. Thus, execution of a query extends the original WDB to WDB-RES. This extension with the set name res as the "entrance point" is the result of the query and can be considered as temporary one until we need this result. (We could also consider update queries which would change the original WDB.)
It was demonstrated in the op. cit. that $\Delta$ covers the expressive power of UnQL and UnCAL from (Buneman et al., 2000) which also have a polynomial time complexity, but do not exhaust the full P-time.

\subsection{Example of Valid Query and the Result of its Execution}

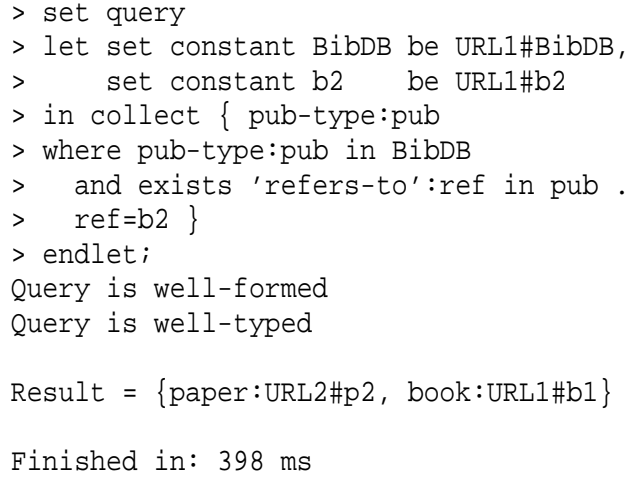

The informal meaning of this query is (imprecisely) as follows: "Find all publications which refer to the book b2." But, as we see, the answer contains, besides the evident publication b1 referring to b2, also p2 which refers to p3 where the latter is formally bisimilar to b2 (same title and author elements), as required in the formal query. If we really want to include only references to the book b2, then seemingly right solution to replace the equality $r e f=b 2$ by the conjunction (ref=b2 and book:ref in BibDB) in the above query does not really help because in any case $\mathrm{p} 3=\mathrm{b} 2$ (are bisimilar) in the above WDB. Equality of (hyper)sets is defined by their elements, elements of elements, etc., i.e. by outgoing-not by incoming_edges. So, after removing redundancies (say, omitting p3) we should have one joint node b2 with two incoming edges BibDB $\stackrel{\text { book }}{\longrightarrow}$ b2 and BibDB $\stackrel{\text { paper }}{\longrightarrow}$ b2 (besides two more incoming refers-to edges from $\mathrm{b} 1$ and $\mathrm{p} 2$ and the evident two outgoing edges). This is probably not what the designer(s) of this distributed WDB had in mind.

This example emphasizes the real meaning of hyperset vs. pure graph approaches to semistructured databases and the role of removing redundancies on the level of the design. The right approach here should be based on a well-chosen discipline:

(i) either to reconstruct this database by replacing labels book and paper by publication and adding outgoing edges from each publication showing its type (' book' or 'paper'; see Figure 3)

(ii) or to use some WDB-schema e.g. requiring that there is only one, up to bisimulation, book/paperedge from BibDB to any given publication. 
"Up to bisimulation" means here that if two children of BibDB are bisimilar then they are labelled by the same label. But it is not our goal here to go into details of such kind of discipline. In any case, we should be precise and accurate in designing the WDB and in formulating both formal and intuitive versions of our queries. The mathematical ground of hyperset theory is quite solid and sufficient for that.

The main point is that if we have a formal query, it has a unique (up to bisimulation) answer-in fact, a hyperset or boolean value - and all the queries are bisimulation-invariant and can be computed in polynomial time (with respect to the size of WDB). Vice versa, any P-time computable and bisimulation invariant (and also "generic" (Lisitsa and Sazonov, 1997)) query is definable in $\Delta$. In fact, this also assumes that the language $\Delta$ has full $\mathrm{P}$-time computable power of restructuring - not only simple retrieval of already existing elements in the WDB. For example the query restructuring the $\mathrm{BibDB}$ database as described in the previous paragraph in (i) could be written in $\Delta$ using the plan performance operator Dec.

\subsection{Example of Restructuring Query}

First, extend the main library by the following useful queries (defined either formally or-for simplicity of presentation-semi-formally):

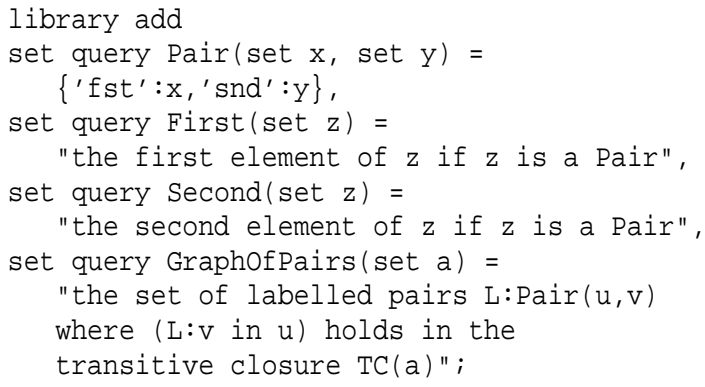

Then the required restructuring query (in (i) above) is defined as follows:

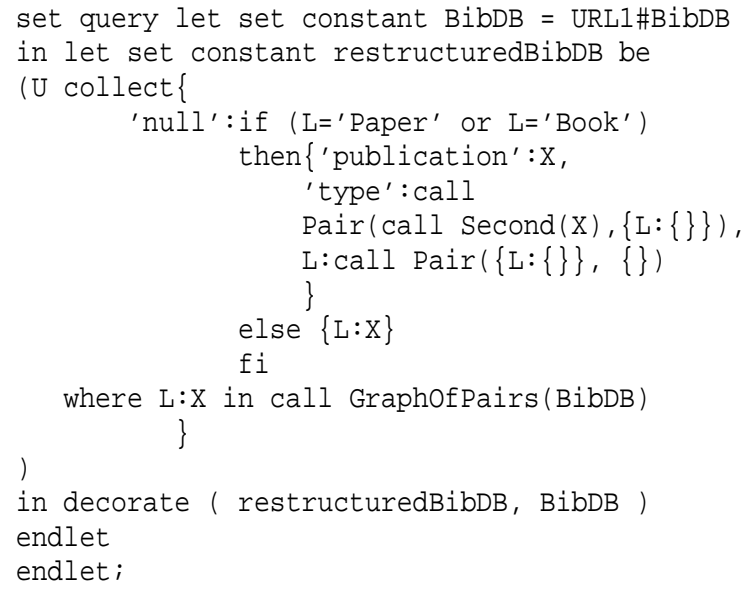

Here 'null' is a label whose value is not important, GraphofPairs (BibDB) is essentially the original bibliography graph in Figure 2 represented in the traditional set theoretic way as the set of (ordered) pairs, and restructuredBibDB denotes the result of the required restructuring of this graph as set of pairs. At last, decorate (restructuredBibDB, $\mathrm{BibDB}$ ) (the plan performance operator applied to the plan-set of pairs restructuredBibDB) essentially transforms this set of pairs understood as a graph into corresponding system of set equations (as it is described in Section 2) with BibDB serving as the main set name. The content of the set BibDB (the result of this query) is a rather lengthy nested bracket expression (a set of sets of sets ...) which is omitted here. The corresponding graph is as follows:

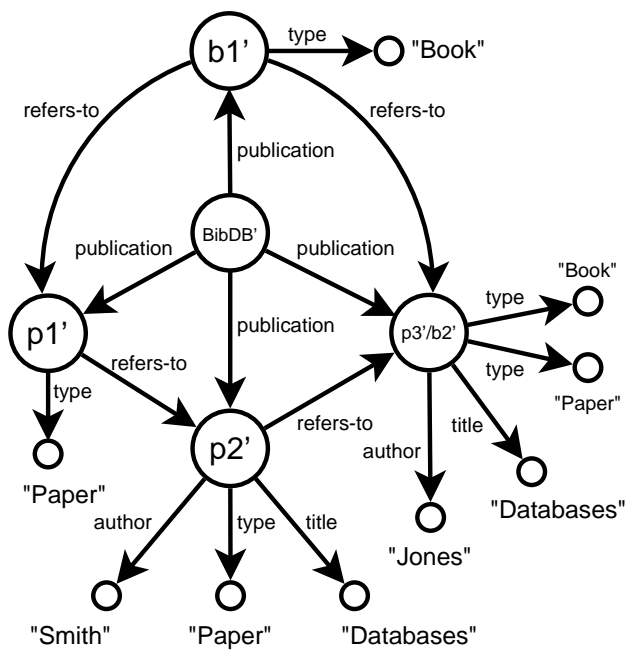

Figure 3: The result of the restructuring query

The fact that one publication has the type both of book and paper is the result of the initial design of BibDB. It is not a failure of the above query.

\section{FURTHER EXTENSIONS}

\subsection{Path Expressions}

The ability to select nodes of a WDB graph to arbitrary depth can be elegantly achieved using path expressions. As shown in (Sazonov, 2006), the action of a rich class of path expressions is definable in the original $\Delta$, itself having no path expressions at all, with the help of TC and Rec. Our next goal is to implement the extension of $\Delta$ by path expressions like in

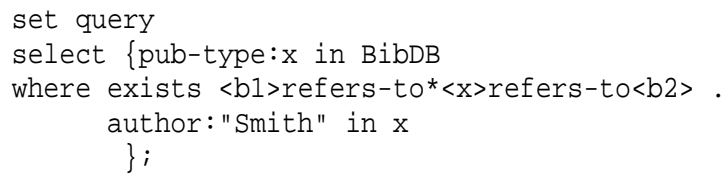


Quantification occurs over paths from b1 to b2 having an appropriate intermediate set/node $x$. Due to p3 $\approx$ b2 the answer to this query is the non-empty set \{paper:p2\}. It would be empty if to remove " $\star$ ".

\subsection{Supporting Bisimulation/Strong Extensionality in Background Time}

One of the key points of our approach is the interpretation of WDB-graph nodes as set names where different nodes can, in principle, denote the same (hyper)set. Whether it is so is defined by the bisimulation relation which can be computed by appropriate (recursive) comparison of labelled child nodes. Thus, in outline, to check bisimulation of two nodes we need to check bisimulation (or non-bisimulation) between some children, granchildren, etc. of the given nodes, i.e. a lot of nodes could be involved. If WDB is distributed, the communication overhead of downloading the relevant XML-WDB files will be too great. (There is also the analogous problem with TC, not discussed here.) So, the equality relation for hypersets seems intractable practically, although theoretically it takes polynomial time with respect to the size of WDB. Nevertheless, we consider that hyperset approach to WDB or semistructured databases is worth to be implemented because it suggests a very clear and mathematically well-understood view on semistructured data and their querying. Thus, the question is whether bisimulation problem can be resolved in any reasonable and practical way. Some possible approaches and views are outline below.

Firstly, should we expect that in reality bisimilar nodes in WDB (i.e. redundancies in WDB) will appear frequently? We could rather assume that WDB is permanently supported in a strongly extensional state i.e. with any rare redundancies eliminated.

Redundancies arising during query execution. As we described above, execution of queries leads to (temporary) extension of the original WDB potentially leading to new redundancies (so that equality subqueries applied to newly generated nodes can be non-trivial). But these redundancies can also be eliminated locally at the server executing the query. Moreover, the algorithm of query execution could be amended in such a way that as soon as a new (auxiliary) nodes/set names are generated (like res, res 1 in Section 4) any possible redundancies will be eliminated immediately.

Let, in general, $\mathrm{WDB}^{\prime}$ be an extension of the given strongly extensional (non-redundant) WDB by a set $N$ of new set names and by new set equations $n=\{\ldots\}$ with the right-hand sides involving both old and new set names. (The original WDB-set equations remain the same in $\mathrm{WDB}^{\prime}$.) Also, without loss of generality and for the sake of the argument we may consider that all old and new set equations are flat-involving no nesting. This is the situation which can arise during computation of a query when new set names and set equations are generated. The question is how to compute new bisimulation relation $\approx^{\prime}$ on $\mathrm{WDB}^{\prime}$ by using the trivial bisimulation relation coinciding with identity $=$ on the original WDB. Evidently, $\approx^{\prime}$ restricted to WDB nodes/set names coincide with $=$ on WDB because set names participating in both parts of set equations of WDB are also from WDB. (WDB remains "self-contained".)

Now, we can conclude from the definition of bisimulation relation that only those nodes from the original WDB are needed to compute $\approx^{\prime}$ (between new nodes and also new vs. old nodes) which are nodes/set names appearing in the new set equations and their children. This restricts the number of downloaded WDB files, and thus elimination of redundancies can be done almost locally.

Redundancies which can appear during a local update of a WDB file are more problematic because the old non-bisimilar nodes outside this file might become bisimilar due to possible links (or paths) to the local nodes with changed/added meaning. The appropriate strategy of removing all such redundancies is not so straightforward (as above) and needs to be developed yet. However, taking into account the locality of changes, this task does not seem to be unrealistic.

Local bisimulation. Assume that all WDB nodes are divided into classes $L_{i}$ according to their sites (WDB servers) or even files. There is a quite natural definition of local (i.e. computed locally; see (Delta-WDB Site, 2007)) lower and upper "best" approximations

$$
n_{1} \approx_{-}^{L} n_{2} \Rightarrow n_{1} \approx n_{2} \Rightarrow n_{1} \approx_{+}^{L} n_{2}
$$

to the global bisimulation relation $\approx$ on the whole WDB. All these local approximations can help to compute and to permanently support global bisimulation in a distributed way in background time. Moreover, we could require "local independency" $\left(\approx_{-}^{L}=\right.$ $\approx_{+}^{L}$, and hence $=\approx\lceil L$ ) and additionally the "local non-redundancy" $\left(\approx_{-}^{L}=\approx_{+}^{L}==^{L}\right)$.

Deliberate redundancies in WDB can also appear, called mirroring in WWW. But if to require that mirroring in WDB should be "officially" registered, then such a deliberate redundancy should most plausibly be dealt with in a quite feasible way.

In general, WDB should not be assumed to be just another version of WWW, freely extensible by anybody in the world. That is, appropriate discipline and restrictions in working with WDB could make the problem of bisimulation practically resolvable. 


\section{COMPARISONS}

The crucial feature of our approach to semistructured databases distinguishing it from others such as Lorel (Abiteboul et al., 1997) and (even the most closest to our approach) UnQL (Buneman et al., 2000) is its (hyper)set theoretical character. Also the query language $\Delta$ has mathematically precise description of its expressive power (as P-time) which makes it sufficiently complete from the theoretical point of view. In this sense our approach is top-down-from theory to practice.

Some important aspects from the practical point of view such as path expressions are currently not included in the language, unlike the approaches from op. cit., however being formally definable in the original $\Delta$ and pending implementation. As to the UnQL language and the related language UnCAL, they were shown to be embeddable in Delta, but not vice versa; see the technical details in (Sazonov, 2006). In a sense the same holds for Lorel. (Here we do not take into account that Lorel was later migrated to the query language to XML, where the order plays an essential role.) The original Lorel deals with graphs, like $\Delta$, but it considers literal equality between graph nodes (oids) instead of using bisimulation relation. Lorel also uses equality between sets of oids, which, however, is not "deep" set equality assumed by bisimulation. Therefore, Lorel would treat our example with BibDB differently, and only very informal comparison is possible. Thus, Lorel can be said to be also strictly embeddable in $\Delta$ (ignoring path variables potentially leading to the exponential complexity and, for simplicity, some aspects like typing and coercion). There is no recursion operator (except Kleene's star in path expressions) and nothing similar to decoration operator (important for deep restructuring) in Lorel. (However StruQL can do something reminding decoration; see e.g. (Abiteboul et al., 2000).) In a sense hyperset $(\Delta)$ vs. graph approaches (UnQL and Lorel) compliment each other. Finally, our implementation assumes distributed WDB, like WWW.

Conclusion. Although $\Delta$ does not include yet path expressions and some other useful explicit constructs of the languages UnQL and Lorel, we already have a working and quite expressive (in a sense more expressive) query language, and this was our current goal. Of course, there is a lot to do for making this a fullfledged and efficient (hyper) Set based WDB Management System (WDBMS or SDBMS).

\section{REFERENCES}

Abiteboul, S., Buneman, P., and Suciu, D. (2000). Data on the Web-From Relations to Semi-structured Data and
$X M L$. Morgan Kaufmann Publishers, San Francisco, California.

Abiteboul, S., Quass, D., McHugh, J., Widom, J., and Wiener, J. L. (1997). The Lorel query language for semistructured data. International Journal on Digital Libraries, 1(1):68-88.

Aczel, P. (1988). Non-Well-Founded Sets. CSLI, Stanford, CA.

Barwise, J. (1975). Admissible Sets and Structures. Springer, Berlin.

Barwise, J. and Moss, L. (1996). Vicious circles: on the mathematics of non-wellfounded phenomena. Center for the Study of Language and Information.

Buneman, P., Fernandez, M., and Suciu, D. (2000). UnQL: a query language and algebra for semistructured data based on structural recursion. The VLDB Journal, 9(1):76-110.

Codd, E. F. (1983). A relational model of data for large shared data banks. Communications of the ACM, 26(1):64-69.

Dahlhaus, E. and Makowsky, J. A. (1986). The choice of programming primitives for SETL-like programming languages. In ESOP'86, LNCS 213, pages 160-172.

Dahlhaus, E. and Makowsky, J. A. (1992). Query languages for hierarchic databases. Information and Computation, 101:1-32.

Delta-WDB Site (2007). http://www.csc.liv.ac.uk/ molyneux/ICSOFT2007appendix/.

Leontjev, A. and Sazonov, V. (2001). $\Delta$ : Set-theoretic query language capturing logspace. Annals of Mathematics and Artificial Intelligence, 33:309-345.

Lisitsa, A. and Sazonov, V. (1999). Linear ordering on graphs, anti-founded sets and polynomial time computability. Theoretical Computer Science, 224(12):173-213.

Lisitsa, A. and Sazonov, V. Y. (1997). Bounded hyperset theory and web-like data bases. In Proceedings of the Kurt Goedel Colloquium (KGC 1997), volume 1234, pages 178-188.

McHugh, J., Abiteboul, S., Goldman, R., Quass, D., and Widom, J. (1997). Lore: A database management system for semistructured data. SIGMOD Record, 26(3):54-66.

Sazonov, V. Y. (1987). Bounded set theory, polynomial computability and $\Delta$-programming. In Lect. Not. Comput. Sci., volume 278, pages 391-397.

Sazonov, V. Y. (1993). Hereditarily-finite sets, data bases and polynomial-time computability. Theoretical Computer Science, 119(1):187-214.

Sazonov, V. Y. (2001). Using agents for concurrent querying of web-like databases via a hyperset-theoretic approach. In PSI '02: 4th International Andrei Ershov Memorial Conference on Perspectives of System Informatics, pages 378-394, London, UK. SpringerVerlag.

Sazonov, V. Y. (2006). Querying hyperset / Web-like databases. Logic Journal of the IGPL, 14(5):785-814. 